\title{
Amplitude Analysis of Frustrated Systems of Coupled Oscillators
}

\author{
Yoko Uwate $^{\dagger}$, Yoshifumi Nishio ${ }^{\dagger}$ and Ruedi Stoop ${ }^{\ddagger}$ \\ $\dagger$ Dept. of Electrical and Electronic Engineering, Tokushima University \\ 2-1 Minami-Josanjima, Tokushima, Japan \\ Email: \{uwate, nishio\}@ee.tokushima-u.ac.jp \\ \$nstitute of Neuroinfomatics, University / ETH Zurich, \\ Winterthurerstrasse 190, CH-8057 Zurich, Switzerland, \\ Email: ruedi@ini.phys.ethz.ch
}

\begin{abstract}
By adding some kinds of frustrations to coupled oscillatory networks, interesting synchronization phenomena can be obtained. In this study, we propose amplitude analysis by using whole power consumption in coupled oscillatory networks with frustrations.
\end{abstract}

\section{Introduction}

Modeling of natural phenomena becomes very important research topic to predict extreme weather consistent with global warming, recently. Coupled oscillatory systems are good models to express essential role of high-dimensional nonlinear phenomena occurring in the field of natural sciences. Therefore, studies of synchronization phenomena have been extensively reported in various areas such as physical [1],[2], biological [3],[4] and electrical [5] systems.

On the other hand, there are several types of polygonal network structures (e.g. Honeycomb structure and crystal structure) in the natural science. Generally, for the studies of large-scale network using coupled oscillators, a ring, a ladder and a two dimensional array structure are often investigated. However, there are not many discussions about coupled polygonal oscillatory networks by using electrical oscillators.

Furthermore, we have been interested in coupled oscillatory networks which has some sort of frustrations of network structure [6],[7]. Because, we consider that frustration have possibility to produce the other types of synchronization such as more complex phenomena and different synchronization from the original system.

In our previous study, synchronization phenomena in coupled polygonal oscillatory networks with frustration was investigated. In this system, odd number of van der Pol oscillators are connected to every corner of polygonal network and frustration is occurred by the shared branch. We have confirmed that the phase difference between the shared oscillators was shifted, then other oscillators synchronized to compensate this phase shift. In order to solve the phase difference in the circuit system, we focused on the power consumption of the coupling resistors in the whole system and proposed the theoretical analysis method by finding the minimum value of the power consumption function. By using computer simulations and theoretical analysis, we confirmed that coupled oscillators tended to synchronize to minimize the power consumption of the whole system [8]. In the proposed theoretical analysis, we use the equal amplitude for all coupled oscillators. However, the accurate amplitude of coupled oscillators has different values by the effect of frustrations.

In this study, we propose amplitude analysis by using the power consumptions of the coupling resistors and the nonlinear resistors in coupled oscillatory networks with frustrations.

\section{Three Coupled Oscillators as a Ring Topology}

\subsection{Circuit Model and Equations}

The circuit model of three coupled van der Pol oscillator as ring topology is shown in Fig. 1.

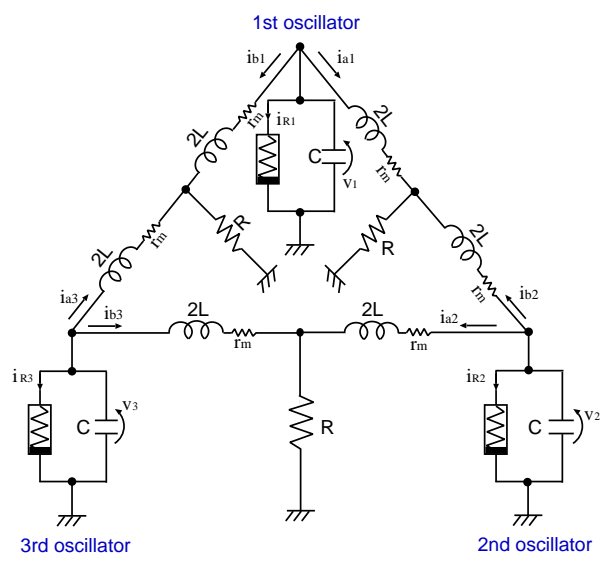

Figure 1: Three coupled van der Pol oscillators as a ring topology.

In the computer simulations, we assume that the $v_{k}-i_{R k}$ characteristics of the nonlinear resistor in each oscillator is given by the following third order polynomial equation

$$
\begin{array}{r}
i_{R k}=-g_{1} v_{k}+g_{3} v_{k}^{3} \quad\left(g_{1}, g_{3}>0\right), \\
(k=1,2,3) .
\end{array}
$$


The normalized circuit equations governing the circuit in Fig. 1 are expressed as [First oscillator]

$$
\left\{\begin{array}{l}
\frac{d x_{1}}{d \tau}=\varepsilon\left(1-\frac{1}{3} x_{1}^{2}\right) x_{1}-\left(y_{a 1}+y_{b 1}\right) \\
\frac{d y_{a 1}}{d \tau}=\frac{1}{2}\left\{x_{1}-\eta y_{a 1}-\beta \gamma\left(y_{a 1}+y_{b 2}\right)\right\} \\
\frac{d y_{b 1}}{d \tau}=\frac{1}{2}\left\{x_{1}-\eta y_{b 1}-\gamma\left(y_{a 3}+y_{b 1}\right)\right\}
\end{array}\right.
$$

[Second oscillator]

$$
\left\{\begin{array}{l}
\frac{d x_{2}}{d \tau}=\varepsilon\left(1-\frac{1}{3} x_{2}^{2}\right) x_{2}-\left(y_{a 2}+y_{b 2}\right) \\
\frac{d y_{a 2}}{d \tau}=\frac{1}{2}\left\{x_{2}-\eta y_{a 2}-\gamma\left(y_{a 2}+y_{b 3}\right)\right\} \\
\frac{d y_{b 2}}{d \tau}=\frac{1}{2}\left\{x_{2}-\eta y_{b 2}-\beta \gamma\left(y_{a 1}+y_{b 2}\right)\right\}
\end{array}\right.
$$

[Third oscillator]

$$
\left\{\begin{aligned}
\frac{d x_{3}}{d \tau} & =\varepsilon\left(1-\frac{1}{3} x_{3}^{2}\right) x_{3}-\left(y_{a 3}+y_{b 3}\right) \\
\frac{d y_{a 3}}{d \tau} & =\frac{1}{2}\left\{x_{3}-\eta y_{a 3}-\gamma\left(y_{a 3}+y_{b 1}\right)\right\} \\
\frac{d y_{b 3}}{d \tau} & =\frac{1}{2}\left\{x_{3}-\eta y_{b 3}-\gamma\left(y_{a 2}+y_{b 3}\right)\right\}
\end{aligned}\right.
$$

where

$$
\begin{gathered}
t=\sqrt{L C} \tau, \quad v_{k}=\sqrt{\frac{g_{1}}{3 g_{3}}} x_{k}, \\
i_{a k}=\sqrt{\frac{g_{1}}{3 g_{3}}} \sqrt{\frac{C}{L}} y_{a k}, \quad i_{b k}=\sqrt{\frac{g_{1}}{3 g_{3}}} \sqrt{\frac{C}{L}} y_{b k}, \\
\varepsilon=g_{1} \sqrt{\frac{L}{C}}, \quad \gamma=R \sqrt{\frac{C}{L}}, \quad \eta=r_{m} \sqrt{\frac{C}{L}},
\end{gathered}
$$

In this equations, $\gamma$ is the coupling strength and $\varepsilon$ denotes the nonlinearity of the oscillators. For the computer simulations, we calculate Eqs. (2)-(4) using a fourth-order RungeKutta method with the step size $h=0.005$. The parameters of this circuit model are fixed as $\varepsilon=0.1, \eta=0.001$. In this circuit system, we observe three-phase synchronization (phase shift: $120^{\circ}$ ).

\subsection{Amplitude Analysis by using Power}

We investigate the amplitude of three coupled oscillators when the coupling strength $\gamma$ is changed. The simulation result is shown in Fig. 2.

We can see that the amplitude is decreased with the coupling strength. How do the amplitude of oscillators determine? In order to solve this question, first, we consider the power of the nonlinear resistor of one van der Pol oscillator without coupling.

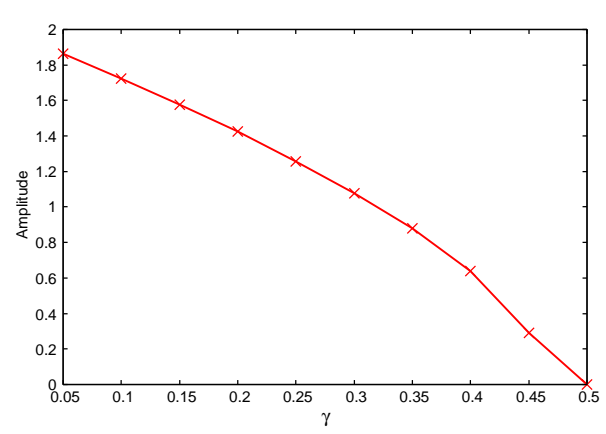

Figure 2: Amplitude of oscillator by changing $\gamma$.

By using the following variable for the current of the nonlinear resistor,

$$
i_{R k}=\sqrt{\frac{g_{1}}{3 g_{3}}} \sqrt{\frac{C}{L}} y_{R k} .
$$

And we assume the voltage of the capacitor as the following equation:

$$
x=A_{m} \sin \tau \text {. }
$$

The power of the nonlinear resistor in van der Pol oscillator is described as Eq. (6).

$$
P_{N R}=\frac{1}{2 \pi} \int_{0}^{2 \pi}\left\{-\varepsilon A_{m}^{2} \sin ^{2} \tau+\frac{1}{3} \varepsilon A_{m}^{4} \sin ^{4} \tau\right\}^{2} d \tau
$$

By solving Eq. (6),

$$
P_{N R}=-\frac{1}{2} \varepsilon A_{m}^{2}+\frac{1}{8} \varepsilon A_{m}^{4} .
$$

Figure 3 shows the graph of Eq. (7) for one van der Pol oscillator. From this figure, we confirm that the amplitude shows \pm 2 when the power is zero. We can see that the amplitude is determined by the balance of the total power (the power of the nonlinear resistors and the coupling resistors) in the circuit system.

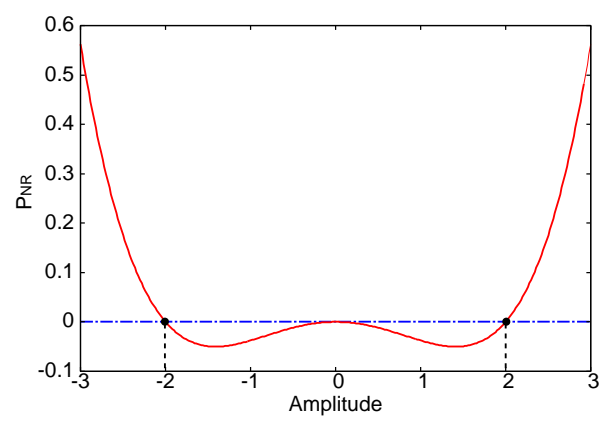

Figure 3: Power of nonlinear resistor.

Next, we apply this approach to three coupled van der Pol oscillators as ring topology. The power of the nonlinear resistor is calculated by using above method (Eq. (7)). In 
order to calculate the power of the coupling resistors, we define the current of the inductor is described as follows.

$$
\left\{\begin{array}{c}
y_{a}=\frac{A_{m}}{2} \sin \left(\tau+\frac{\pi}{2}\right) \\
y_{b}=\frac{A_{m}}{2} \sin \left(\tau+\frac{5 \pi}{6}\right)
\end{array}\right.
$$

The power of the coupling resistor is expressed by Eq. (8).

$$
P_{C R}=\frac{\gamma}{2 \pi} \int_{0}^{2 \pi}\left\{\frac { A _ { m } } { 2 } \operatorname { s i n } ^ { 2 } \left(\tau+\frac{\pi}{2}+\frac{A_{m}}{2} \sin ^{2}\left(\tau+\frac{5 \pi}{6}\right\} d \tau\right.\right.
$$

By solving Eq. (8),

$$
P_{C R}=-\frac{1}{8} \gamma A_{m}^{2}
$$

The total power is obtained by calculating the following equation.

$$
P_{A L L}=3 P_{N R}+3 P_{C R} .
$$

Figure 4 shows the graph of Eq. (10) when the coupling strength is fixed with $\gamma=0.1$. In this case, the amplitude of the oscillators denotes 1.73 when the total power shows zero. This result matches very well with the computer simulation result.

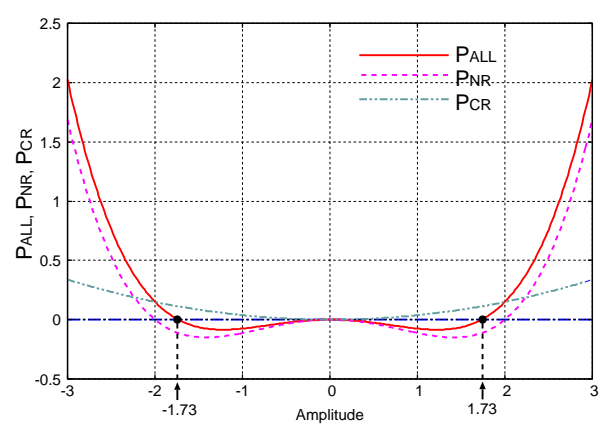

Figure 4: Total power of three coupled van der Pol oscillators as ring topology.

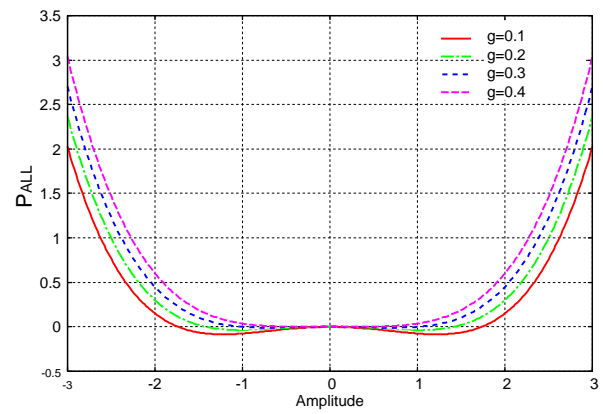

Figure 5: Total power by changing $\gamma$.

Next, we show the graph of total power when the coupling strength $\gamma$ is changed as shown in Fig. 5. By increasing the value of $\gamma$, the amplitude of the oscillators decreases. Figure 6 shows the change of the amplitude with the coupling strength obtained from the theoretical analysis and the computer simulation. From this figure, we can see that the both methods match well when the coupling strength is small.

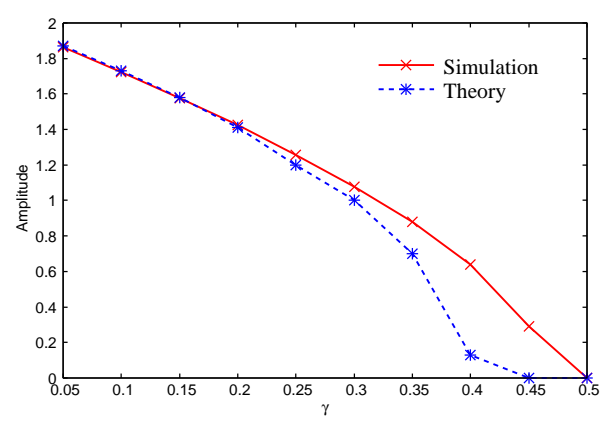

Figure 6: Amplitude of oscillator by changing $\gamma$.

\section{Two Coupled Polygonal Networks}

In this section, we apply the theoretical analysis to find the amplitude by using total power for two coupled polygonal oscillatory networks. The conceptual circuit model of 5-5 coupled networks is shown in Fig. 7.

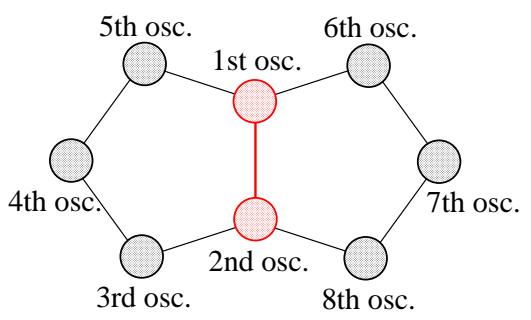

Figure 7: Two coupled odd-number-node polygonal oscillatory networks (5-5 coupled networks).

Table I summarizes the obtained amplitude by using computer simulation when the coupling strength is fixed with $\gamma=0.1$. We confirm that the amplitude of the shared oscillators is smaller value than the other combination oscillators. Namely, we define two amplitudes $A_{m}$ and $B_{m}$ for the two coupled polygonal networks.

Table 1: Amplitude of 5-5 coupled networks (computer simulation)

\begin{tabular}{c|c}
\hline \hline Shared (1st-2nd) osc. & Other combi. \\
\hline \hline 1.933 & 1.986 \\
\hline
\end{tabular}

The power of the nonlinear resistor is obtained by Eqs. (11), (12).

$$
\left\{\begin{array}{l}
P_{N R a}=-\frac{1}{2} \varepsilon A_{m}^{2}+\frac{1}{8} \varepsilon A_{m}^{4} \\
P_{N R b}=-\frac{1}{2} \varepsilon B_{m}^{2}+\frac{1}{8} \varepsilon B_{m}^{4}
\end{array}\right.
$$


The power of the coupling resistor is obtained by Eqs. (13)-(15).

$$
\left\{\begin{array}{l}
P_{C R a}=-\frac{1}{18} \gamma A_{m}^{2}\{\sin (4 \pi+\theta)-\sin \theta-\pi \cos \theta\} \\
P_{C R a b}=-\frac{1}{18} \gamma A_{m} B_{m}\{\sin (4 \pi+\theta)-\sin \theta-\pi \cos \theta\} \\
P_{C R b}=-\frac{1}{18} \gamma B_{m}^{2}\{\sin (4 \pi+\theta)-\sin \theta-\pi \cos \theta\}
\end{array}\right.
$$

The total power is obtained by calculating the following equation.

$$
P_{A L L}=2 P_{N R a}+6 P_{N R a}+P_{C R a}+4 P_{C R a b}+4 P_{C R b} .
$$

Figures 8 and 9 show the graph of the total power for 5-5 coupled networks. In Fig. 8, the amplitude $B_{m}$ is set to 1.98 which is obtained from the simulations. While, in Fig. 9, the amplitude $A_{m}$ is set to 1.90 similary. From these figures, we obtain the amplitudes $A_{m}, B_{m}$ when the graph of total power shows zero.

Finally, the amplitude obtained from theoretical analysis is summarized in Table II. We can see that these amplitudes are very similar to the simulation results (see Table I).

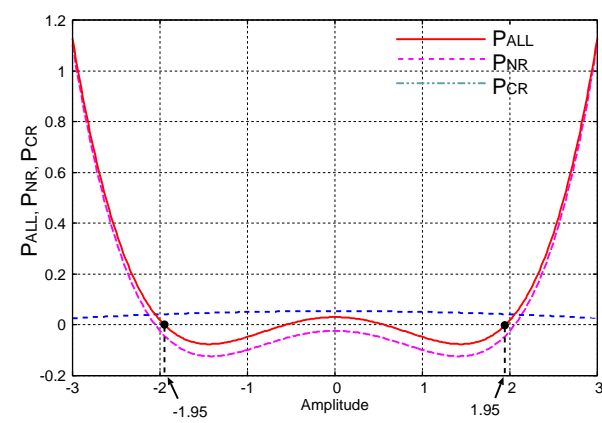

Figure 8: Total power of 5-5 coupled network $\left(A_{m}\right)$.

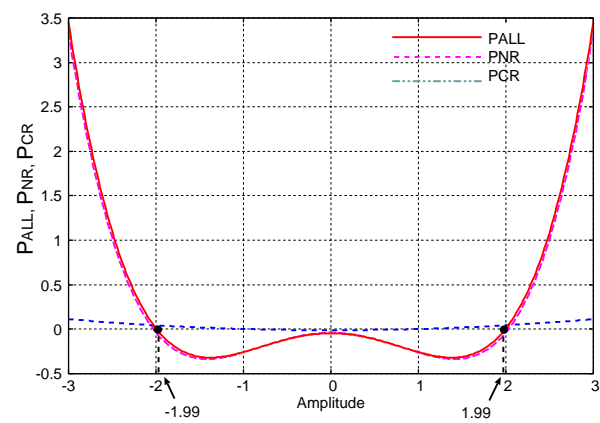

Figure 9: Total power of 5-5 coupled network $\left(B_{m}\right)$.

\section{Conclusions}

In this study, we have proposed amplitude analysis by using whole power consumption in coupled oscillatory networks with frustrations. By using computer simulations,
Table 2: Amplitude of 5-5 coupled networks (theoretical result)

\begin{tabular}{c|c}
\hline \hline Shared (1st-2nd) osc. & Other combi. \\
\hline \hline 1.95 & 1.99 \\
\hline
\end{tabular}

we have confirmed that the amplitude of coupled oscillators is decided by the balance of whole power consumption in the circuit system. Furthermore, we have proposed the theoretical analysis to solve the amplitude of oscillators by finding zero value of the total power functions.

For the future work, we develop a efficiently method to find the minimum value of the power function for the large scale complex networks with many variables. Investigation of synchronization phenomena in coupled polygonal oscillatory systems with strong nonlinearity or in coupled polygonal chaotic oscillators is also our future work.

\section{Acknowledgments}

This work was partly supported by JSPS Grant-in-Aid for Young Scientists 23700269.

\section{References}

[1] G. Abramson, V.M. Kenkre and A.R. Bishop, "Analytic solutions for nonlinear waves in coupled reacting systems," Physica A: Statistical Mechanics and its Applications, vol.305, nos.3-4, pp.427-436, Mar. 2002.

[2] I. Belykh, M. Hasler, M. Lauret and H. Nijmeijer, "Synchronization and graph topology," Int. J. Bifurcation and Chaos, vol.15, no.11, pp.3423-3433, Nov. 2005.

[3] J. Cosp, J. Madrenas, E. Alarcon, E. Vidal and G. Villar, "Synchronization of nonlinear electronic oscillators for neural computation," IEEE Trans. Neural Networks, vol.15, no.5, pp.1315-1327, Sep. 2004.

[4] R. Stoop and C. Wagner, "Neocortex's architecture optimizes computation, information transfer and synchronizability, at given total connection length," International Journal of Bifurcation and Chaos, vol.17, no.7, pp.22572279, 2007.

[5] H.B. Fotsina and J. Daafouza, "Adaptive synchronization of uncertain chaotic colpitts oscillators based on parameter identification" Physics Letters A, vol.339, pp.304-315, May. 2005.

[6] Y. Uwate, Y. Nishio and R. Stoop, "Synchronization in three coupled van der Pol oscillators with different coupling strength," Proc. of NCSP'10, pp. 109-112, Mar. 2010.

[7] Y. Uwate, Y. Nishio and R. Stoop, "Synchronization in Two Polygonal Oscillatory Networks Sharing a Branch," Proc. of NDES'10, pp. 62-65, May 2010.

[8] Y. Uwate and Y. Nishio, "Synchronization in Several Types of Coupled Polygonal Oscillatory Networks," IEEE Transactions on Circuits and Systems I, vol. 59, no. 5, pp. 10421050, May 2012. 